\title{
La naturaleza jurídica de las wikis en el derecho de autor
}

\author{
The legal nature of wikis in copyright
}

\author{
MARCO CORREA PÉREZ \\ Wikimedia, Chile
}

\begin{abstract}
RESUMEN Las wikis, cuyo principal referente es Wikipedia, son herramientas en línea que permiten la colaboración simultánea de múltiples usuarios, dando origen a una producción intelectual que reúne las características de una obra protegida por el derecho de autor. Sin embargo, la doctrina ha dicho poco respecto a la regulación de las wikis, lo que resulta relevante atendidas sus peculiaridades en comparación a las obras literarias clásicas. Este ensayo examina la wiki desde el derecho de autor, tanto nacional como comparado, intentando establecer la naturaleza jurídica de la plataforma y la posibilidad de protección de los distintos tipos de wiki. En ese contexto, también se realiza un inédito análisis doctrinal respecto a la obra colectiva en la Ley de Propiedad Intelectual chilena.
\end{abstract}

PALABRAS CLAVE Derecho de autor, naturaleza jurídica, colaboración, wiki, Wikipedia.

ABSTRACT Wikis, whose main point of reference is Wikipedia, are online tools that allow simultaneous collaboration from multiple users, creating an intellectual product that qualifies as a copyrighted work. However, doctrine has said little with respect to the regulation of wikis, which is 
relevant due to its uniqueness compared with classic literary works. This essay examines the wiki regarding copyright, both national and comparative, attempting to establish the legal nature of the platform and the possibility to protect different types of wiki. In this context, this essay also incorporates an unprecedented doctrinal analysis of the collective work in Chilean Intellectual Property Law.

KEYWORDS Copyright, legal nature, collaboration, wiki, Wikipedia.

\section{INTRODUCCIÓN}

Todo quien esté familiarizado con Internet — que a estas alturas es la mayoría de la población- debe haber usado alguna vez, o al menos conoce aunque sea de nombre, a Wikipedia, una enciclopedia de contenido libre y disponible en más de 280 idiomas, que ha logrado desplazar a otras enciclopedias de formato tradicional, como la prestigiosa Enciclopedia Británica, y que sepultó a su par comercial, la Encarta de Microsoft.

La principal particularidad de Wikipedia, y también la clave de su éxito, es que sus contenidos son construidos, mejorados y actualizados por miles de voluntarios alrededor del mundo, quienes editan simultáneamente desde sus computadoras. Wikipedia es un ejemplo de wiki, una plataforma colaborativa creada en I994 por Ward Cunningham (cf. Cunningham y Leuf, 2004: I4-5), y que además de ser usada por Wikipedia, también lo es por cientos de otros tipos de sitios web, incluyendo redes privadas y entornos corporativos (intranets).

Si bien la doctrina nacional y comparada ha realizado diversos estudios sobre la naturaleza jurídica de obras surgidas en el entorno digital, las wikis han sido particularmente ignoradas por nuestros autores. La mayoría de los análisis se han gestado en el derecho anglosajón y sólo unos pocos han escudriñado el tema dentro de Latinoamérica.

El estudio que proponemos resulta relevante debido a que las wikis son una herramienta que no sólo es de uso masivo en la actualidad, sino que tiene gran proyección futura, en atención al cambio de paradigma en la generación de contenidos, que ha pasado desde una relación jerárquica y unilateral entre los autores y el público consumidor, a una relación simétrica y bilateral, donde todos somos creadores de contenido. El derecho de autor clásico amenaza con obstaculizar, o a lo menos hacer más 
difícil, este cambio de paradigma, cuestión que queremos ejemplificar a través de las wikis.

De todo lo anterior surge nuestro interés en estudiar a las wikis desde el derecho de autor, intentando establecer su naturaleza jurídica mediante su comparación con las obras tipo que nuestra legislación y otras similares han establecido tanto doctrinal como jurisprudencialmente.

\section{LAS WIKIS}

\section{CONCEPTO}

Wiki es una tecnología que permite la colaboración en una página web por usuarios que acceden simultáneamente a ella mediante un navegador de Internet (Sereno, 2010: 210; Charman y Holloway, 2006). Se le identifica como una de las herramientas de producción textual de colaboración «abierta», en contraste a las de colaboración «cerrada», como blogs, foros y comunidades virtuales, cuyos usuarios son limitados por sistemas de registro.

Esta plataforma tiene, por regla general, las siguientes características:

- Dinamismo: Son plataformas cuyo contenido es dinámico, ya que va mutando conforme los usuarios realizan sus contribuciones.

- Simpleza: El diseño de la wiki es simple, con tal de favorecer la colaboración por usuarios que no tengan mayor experiencia en edición web (Sereno, 20I0: 2II).

- Código wiki: Como consecuencia de lo anterior, se utiliza un código fuente especial, conocido como lenguaje wiki, que es más sencillo que el HTML, ocupado por la mayoría de las páginas web (Lamb, 2004: 38).

- Control reducido: Los mecanismos de control, tanto de acceso como de edición, son menos estrictos que en otras plataformas.

Las wikis funcionan sobre la base de un software wiki, que es instalado como un script («archivo de órdenes») en un servidor (Ebersbach, Glaser y Heigl, 2005: I6). El software wiki más popular es MediaWiki, software de código abierto que es utilizado por Wikipedia y otras miles de wikis. 
La interfaz de las wikis está dada fundamentalmente por páginas principales, «entradas» o «artículos», en los que se desarrolla el contenido propio de la wiki. Cada entrada posee un «historial», que es la cronología de cambios realizados al artículo, en donde se registran datos como el tiempo de la edición, el usuario que la hizo e incluso la cantidad de caracteres; y una «discusión», en la que los usuarios pueden discutir el contenido del artículo.

La entrada de wiki puede estar estructurada de dos maneras: un «modo documento» (document mode), en el cual los contenidos están ordenados a la manera de un ensayo o artículo, donde las contribuciones de una persona se confunden con los aportes de las demás; y un «modo hilado» (thread mode), en el que los contenidos son añadidos en estricto orden cronológico, y son fácilmente individualizables, ya que por lo general están acompañados de la «firma» de la persona a quien corresponden. La regla general en las wikis es el uso del «modo documento», ya que fomenta la colaboración en vez de valorar los aportes individuales. Incluso se plantea que el «modo hilado» es ideal para herramientas distintas a la wiki, como foros o grupos de noticias (Cunningham y Leuf, 2004: 3I).

\section{SUJETOS INVOLUCRADOS}

Hay dos clases principales de sujetos que intervienen en una wiki. En primer lugar, y como es lógico en un escenario de web 2.0, está la categoría de los usuarios, contribuyentes o editores de la wiki, quienes son las personas que se dedican a interactuar de manera directa con los contenidos alojados en dicha plataforma, mediante la creación, edición y organización del material.

El otro tipo de sujeto involucrado es llamado genéricamente administrador, el que ha sido definido como «quien (o quienes) provee el software colaborativo - la wiki propiamente dicha-, su desarrollo y otras tareas especializadas relacionadas con el mantenimiento y mejoramiento de la tecnología, y el sitio wiki» (Sereno, 20I0: 2I3).

Sin embargo, esta descripción tiende a ser demasiado amplia, puesto que, en primer lugar, es una categoría que agrupa funciones que pueden ser ejercidas por distintas personas y que implican diversos niveles de derechos y responsabilidades respecto al contenido de la wiki; y, 
en segundo lugar, porque hay editores de una wiki que pueden realizar tareas de mantenimiento y administración sin necesariamente proveer o desarrollar el software wiki a la página, generándose híbridos usuario-administrador —conocidos como «burócratas» (o «bibliotecarios») y "operadores del sistema» (system operators o sysop) (Snake-Beings, 20I0: I4) - que complicarían el análisis posterior. Por ello, preferimos descomponer las funciones «administrativas» de una wiki en tres sujetos (Black, Delaney y Fitzgerald, 2007: 247):

- El propietario de la wiki o administrador propiamente tal, es decir, la persona (natural o jurídica) que posee el nombre de dominio y además es creador y operador de la wiki; en el caso de Wikipedia, la propietaria sería la Wikimedia Foundation Inc.

- Los proveedores de la tecnología (software) wiki, por ejemplo, Wikia es proveedora de la plataforma wiki a un centenar de sitios wiki de diversas temáticas.

- Los proveedores de alojamiento (hosting) de la wiki.

Naturalmente, dos o más de las categorías dadas pueden radicarse en una misma persona, lo que variará caso a caso. Además, a estos sujetos podrían sumarse eventualmente otros, como los proveedores de acceso a Internet. Sin embargo, las categorías que más nos importarán a efectos de este estudio serán las de usuarios y propietarios de la wiki.

TIPOS DE WIKI

La práctica demuestra que el formato wiki es flexible, y que, dependiendo del uso al cual esté destinado, éste puede tomar formas muy diversas. Por ello, estableceremos los distintos parámetros que puede adoptar una wiki.

En cuanto a sus contenidos, se debe hacer una diferencia entre los internos, originales o autogestionados, los cuales se generan en la misma wiki y sin su soporte no existirían, y los externos o previamente existentes, que se incorporan a una wiki, para conformar un catálogo de obras.

En cuanto al acceso a dichos contenidos, las wikis pueden ser: públicas, es decir, cualquier persona podrá acceder a los contenidos, sin necesidad de pertenecer a un registro de usuarios; y privadas, las cuales no permiten que cualquier persona pueda acceder a los datos de la wiki, 
como es el caso de las wikis de carácter corporativo. Si bien el carácter privado contradice en buena medida nuestra definición de «colaboración abierta» de la wiki, dichas páginas son aceptadas como wikis debido a que operan con el software wiki y, en una interpretación muy extensiva del concepto, el contenido estaría «abierto» a sus usuarios selectivamente escogidos.

En cuanto a la edición de los contenidos de una wiki, éstas pueden ser: libres, aquellas que permiten que cualquier usuario, registrado o no, pueda editar los contenidos de la wiki en su integridad; y restringidas, las cuales podrán ser editadas sólo por los usuarios que posean una cuenta de usuario en la wiki.

Relacionado con el parámetro anterior, está la clasificación según el control de contenidos de la wiki. Ésta apunta al rol que tiene en la wiki su propietario, respecto al ejercicio de vigilancia y censura de los contenidos que en ella existen. Dicho rol puede ser: activo, en la que el propietario decide qué contenidos se incorporarán en la wiki y cuáles no, y pasivo, en las cuales el propietario no interviene en dichas materias, y por regla general las entrega a la comunidad de colaboradores de la wiki. Hay quienes plantean que el modelo de wiki debería ir variando hacia la selectividad en la edición, a diferencia de la «anarquía feliz» de las wikis de control pasivo, ya que supuestamente se aseguraría una mayor confiabilidad en los contenidos (cf. Farley, 2007: 253). Sin duda, estamos en desacuerdo con dicha concepción, ya que ello implicaría una contradicción al carácter colaborativo de la plataforma.

La aplicación de dichos parámetros da origen a tres formas principales de wiki:

- Wikis libres o propiamente tales: Acceso público, edición libre, control pasivo.

- Wikis mixtas: Acceso público, edición restringida, control activo.

- Wikis cerradas: Acceso privado, edición restringida, control activo.

El paradigma que más representa al concepto de wiki es la wiki libre, dado que es la que más fomenta la colaboración masiva multiautoral; así, Cunningham y Leuf definen genéricamente a las wikis bajo los parámetros de las wikis libres: 
La wiki es inherentemente democrática: cada usuario tiene exactamente las mismas capacidades que cualquier otro usuario. Permite la colaboración web sin tener que lidiar con cuentas y contraseñas. A pesar que a simple vista ello puede parecer una forma extremadamente riesgosa de manejar datos modificables, la experiencia muestra que en la práctica se provoca poco daño al contenido de la wiki incluso en ausencia de mecanismos de seguridad (2004: I 7). ${ }^{1}$

\section{LA WIKI COMO OBRA}

El Convenio de Berna establece en su artículo segundo que dentro de las obras protegidas están las "producciones en el campo literario, científico y artístico, cualquiera que sea el modo o forma de expresión». Resulta claro que la creación de contenidos en el contexto de una wiki es una producción intelectual, siendo las ediciones en la plataforma wiki (digitales, por regla general escritas) su forma de expresión.

La norma antes citada da origen a tres categorías de obra: literarias, científicas y artísticas. ¿A cuál de ellas corresponde la wiki? Creemos que lo más acertado es denominar a las wikis como obras literarias, en el entendido que este concepto no está restringido a las obras de la literatura, sino que aplica «a todas las formas escritas originales, sean de carácter literario, científico, técnico o meramente práctico, prescindiendo de su valor o finalidad» (Herrera, I 999: I 5). Así, el carácter flexible de la wiki permite que ésta sirva no sólo para crear una enciclopedia — como Wikipedia- o una wikinovela (obra literaria en el sentido estricto), sino que también es útil como manual técnico (obra «científica»), o incluso como un catálogo de obras artísticas (fotografías, reproducciones de pinturas, dibujos, grabados, etcétera).

Lo anterior permite plantar que el concepto de obra forjado por el derecho de autor clásico se ve sobrepasado ante nuevos tipos de obras que, como la wiki, tengan un carácter flexible que no puede ser fácilmente adaptado a los modelos tradicionales, y que por su naturaleza de producción intelectual multiautoral rompe con los esquemas del autor individual (cf. Chon, I996).

I. Todas las traducciones son nuestras, salvo que se indique lo contrario. 
La ley de derechos de autor está mal ajustada a la cooperación entre grandes grupos de creadores dispersos. Esto es cierto para todos los sistemas legales nacionales que están históricamente organizados sobre la idea de una única entidad creativa centralizada (una persona o una corporación). [...] Al final, la ley es incapaz de considerar la idea del conocimiento compartido (Wielsch, 2010: 103).

Habiendo establecido que, bajo un concepto amplio del término, la wiki es una obra «literaria», ahora cabe preguntarse, ¿bajo qué reglas se regula? Considerando sus peculiares características, esta última pregunta nos obliga a realizar un estudio pormenorizado de esta herramienta bajo el lente del derecho de autor. En lo sucesivo nos propondremos calificar jurídicamente a las wikis, en primer lugar como una obra en sí misma, y posteriormente como un compendio de múltiples obras.

\section{LA WIKI COMO OBRA EN SÍ MISMA}

\section{OBRAS EN COAUTORÍA}

Dada la naturaleza de las wikis, en las cuales intervienen múltiples usuarios, se ha planteado si éstas podrían encajar en las obras en coautoría, que en definición de Lipszyc son aquellas donde «varios autores contribuyeron a la creación de una obra trabajando juntos, o bien por separado pero creando sus aportes, del mismo o de diferente género, para que sean explotados en conjunto y formen una unidad» (I993: I 29). Dentro de esta categoría están las obras en colaboración (en derecho anglosajón, joint autorship) y las obras colectivas (collective work), las cuales analizaremos a continuación.

\subsection{OBRA EN COLABORACIÓN}

La doctrina define las obras en colaboración como aquellas «creadas por dos o más personas que trabajan juntas, o al menos tienen mutuamente en cuenta sus contribuciones y bajo una inspiración común» (Lipszyc, I993: I30). Este concepto puede ser visto desde dos concepciones: una amplia, en donde «si bien las contribuciones de los diferentes creadores son individualizables, entre ellos ha habido acuerdo respecto de la forma en que aportarían sus partes a la obra global y éstas aparecen ligadas 
por una comunidad de inspiración»; y una concepción estricta, la cual se verifica "cuando los coautores han trabajado juntos con un grado de compenetración y en forma tal que, una vez concluida la obra, resulta imposible determinar cuál es la parte atribuible a cada uno de ellos» (Lipszyc, I993: I30-I).

La legislación chilena define a la obra en colaboración en el artículo 5 letra b) de la Ley I7.336 de Propiedad Intelectual (en adelante, «LPI») como la «producida, conjuntamente, por dos o más personas naturales cuyos aportes no puedan ser separados». En la clasificación doctrinal, esta definición se circunscribe a la concepción estricta. La legislación de copyright de los Estados Unidos define la obra en colaboración como aquella «preparada por dos o más autores con la intención de que sus contribuciones estén fundidas en las partes inseparables o interdependientes de un todo unitario». ${ }^{2}$ La definición anterior, en cambio, da lugar a una interpretación amplia.

La legislación británica define la obra en colaboración en similares términos a la ley chilena. ${ }^{3}$ Por ello, resulta pertinente recoger el test que la jurisprudencia británica ha elaborado para verificar la autoría en colaboración. La sentencia del caso Hadley and Others v. Kemp and Another repasa los requisitos de la obra en colaboración que han establecido las cortes del Reino Unido. Éstos son (Simone, 20I I):

- Los coautores deben haber aportado una cantidad considerable de contenidos originales a la obra: Esto no significa que los aportes sean iguales a los de sus colaboradores en términos de cantidad, calidad u originalidad — según lo establece la sentencia de Stuart v. Barrett-, sin embargo, «sería sorprendente si una contribución menor fuese suficiente para que una persona fuera coautora y así convertirla en propietaria al igual que otra u otras personas que hayan contribuido mucho más que ésta». ${ }^{4}$

2. Copyright Law of the United States, título I 7 del US Code. Su sección Io I establece: "A 'joint work' is a work prepared by two or more authors with the intention that their contributions be merged into inseparable or interdependent parts of a unitary whole».

3. Copyright, Designs and Patents Act I988. Su sección ro establece que la obra en colaboración es «a work produced by the collaboration of two or more authors in which the contribution of each author is not distinct from that of the other author or authors».

4. Hadley and Others v. Kemp and Another, pág. 32. 
- El aporte debe haber sido realizado en cumplimiento de algún diseño común o colaboración: En el caso Thomas Morison QC in Stuart v. Barrett se describe el proceso de creación de una canción por un grupo musical, en donde "alguien empieza a tocar y el resto [de la banda] se le une e improvisan y mejoran la idea original. La pieza final fue, sin duda, el producto de las aptitudes compositoras en colaboración de los miembros del grupo». ${ }^{5}$

- Que no haya divisibilidad de los aportes: Las contribuciones no deben estar separadas o ser distinguibles una de otra. El caso Robin Ray v. Classic FM PLC aborda este requisito cuando establece que no es suficiente que haya existido una división de labores entre dos personas en el proyecto de escribir un libro, si la responsabilidad y autoría de éste sólo recae en una de ellas. ${ }^{6}$

Este test, como puede apreciarse, tiene criterios más fácticos que jurídicos (Black, Delaney y Fitzgerald, 2007: 252), por ello, las circunstancias de cada caso (tipo de obra, rol de los posibles autores) determinarán si puede haber autoría en colaboración.

Los puntos más conflictivos para que las wikis pasen este control son los requisitos primero y segundo. El tercer punto, respecto a la separabilidad de los aportes de una wiki, está relativamente zanjado por la doctrina. Charman y Holloway (2006) desechan la divisibilidad de las contribuciones en wikis, debido a que éstas se tornan tan difusas, que se hace imposible — al menos en términos prácticos- poder distinguir entre el aporte de una persona y el de otra al mirar una entrada.

A pesar de que la mayoría de las wikis tiene un registro de las ediciones realizadas en la sección «historial», esta estructura no es la que justamente hace más evidente la importancia de los aportes individuales (Garon, 20I0: IOO), ya que la entrada presenta los contenidos al público como un todo que perfectamente podría haber sido hecho por una sola persona.

Las wikis, por su estructura y wikietiqueta, reducen la identidad de los contribuyentes a los historiales de las páginas. Mientras que es probable que los miembros de una comunidad wiki activa conozcan los

5. Hadley and Others v. Kemp and Another, pág. 3 I.

6. Hadley and Others v. Kemp and Another, pág. 33. 
nombres o apodos de los contribuyentes más frecuentes, no lo es para el público general. Los autores de una wiki son esencialmente anónimos. El popular formato de blog, en cambio, tiende a enfatizar el nombre del autor y le entrega a éste mucho más control sobre el contenido (Garon, 2010: 107).

Debemos, entonces, analizar los otros dos requisitos en el caso de las wikis. Respecto al primer factor del test de una obra en colaboración, que se refiere a la consistencia del aporte, es discutible que se verifique en las wikis, dependiendo mucho de los casos en concreto. Así, podría darse el caso de una cantidad muy restringida de usuarios que escriben todos los contenidos en una wiki cerrada, aportando entradas completas o secciones sustanciales de éstas. No obstante, la naturaleza de la wiki, como ya lo hemos explicado, es que cualquier aporte, independientemente a la cantidad de caracteres que éste involucre, es valorable, incluso si se trata de la corrección de una letra, o de añadir un signo de puntuación.

El segundo paso del test implica determinar si las wikis son creadas por un trabajo mancomunado y con una inspiración y diseño común. En este punto, la respuesta es más categórica que en el caso del primer requisito.

El actuar de los usuarios de plataformas de Internet por lo general es autónomo, ya que resulta complejo que se genere una instancia para ponerse de acuerdo con sus pares respecto a eventuales creaciones.

Así pues, en un boletín board, o news o en un chat, su participación activa colabora en un total, que en su conjunto puede tener una originalidad, con independencia de las partes, y en donde los posibles 'coautores' ni se conocen ni se han puesto de acuerdo en tal creación. Y ni qué decir tiene de aquellas ocasiones en que se requiere a los usuarios o visitantes de una web su participación para la creación de una determinada obra o sin requerimiento hay espontánea participación (García, 2005: 60).

En el caso de las wikis, dicha tendencia se mantiene. Black, Delaney y Fitzgerald (2007: 252) han planteado que es a lo menos debatible pensar que la edición en wikis sea análoga a la corrección, edición o compilación conjunta que se realiza para dar lugar a una obra en colaboración.

Para ejemplificar su posición, los autores citan el caso Samuelson v. Producers' Distributing Co, Ltd., donde un dramaturgo demandó in- 
demnización de perjuicios porque en la adaptación cinematográfica de una de sus obras se le otorgó la coautoría a un actor que la había interpretado teatralmente, George Clarke. En este caso, a pesar de que Clarke pudo añadir frases a la obra mediante la improvisación, y que esas frases fueron usadas en la película basada en la obra, no existe obra en colaboración entre el dramaturgo y el comediante. El juez Romer argumentó que «el autor de una obra puede tener el derecho de autor, y tiene el derecho de autor, sobre la obra como un todo, sin tener los derechos de autor o ser el autor de cada simple frase de la obra». ${ }^{7}$

Para que haya colaboración efectiva, los individuos deben actuar conjuntamente, a diferencia de lo que ocurrió en el caso Samuelson, donde el comediante se limitó a añadir frases a la obra del demandante. Cosa similar ocurre en las wikis, donde un usuario modifica los contenidos aportados por un tercero, sin existir un trabajo asociado entre ambos.

Sereno dice que si bien existen las páginas de «discusión» en cada entrada de una wiki, donde los usuarios pueden debatir aspectos de ésta, la práctica muestra que dichas herramientas no permiten hablar de una verdadera planificación común de los contribuyentes, pues muchas veces dichas discusiones estarán cargadas de un «laconismo extremo» (2010: 227).

Si bien Sereno puede pecar de exageración al hablar de laconismo, considerando que hay casos excepcionales en que usuarios de una wiki actúan coordinadamente (o intentan hacerlo) para lograr un producto, la verdad es que por regla general cada editor realiza sus contribuciones de manera independiente (y muchas veces oponiéndose a los aportes anteriores). Constancia de aquello son las «guerras de ediciones», en donde dos o más usuarios modifican entre sí las ediciones de otros, debido a discrepancias de criterio respecto a los contenidos. La mayor parte de las veces estas «guerras» no se resuelven por un consenso entre los involucrados, sino que por la intervención de un tercer usuario con facultades de sysop o bibliotecario, que pueda bloquear los contenidos, o a los usuarios.

Otro factor que desincentiva la creación bajo una inspiración común en wikis es que la mayoría de los contribuyentes no se conoce personalmente, salvo por un pequeño núcleo de editores frecuentes que incluso

7. Samuelson v. Producers' Distributing Co, Ltd., p. 8. 
llegan a entablar relaciones offline. Como excepción están las wikis corporativas, en las cuales la mayoría de los usuarios son empleados de una compañía y se conocen entre sí.

Por último, cabe señalar la inconveniencia de que una wiki fuera considerada obra en colaboración con respecto a su plazo de protección. Como en este tipo de obras, el plazo de protección post mortem auctoris se considera «desde la muerte del último coautor» según el artículo I 2 de la LPI, podría darse el absurdo de que una wiki activa nunca cayera en el dominio público, pues cada nuevo usuario que interviniera en ella aumentaría el plazo de protección de ésta ad infinitum.

Los antecedentes expuestos en razón del análisis hecho por la jurisprudencia norteamericana en relación a las obras en colaboración, nos permiten descartar que las wikis sean clasificables en dicho tipo de obra. Dicha conclusión, basada en jurisprudencia del common law, es reafirmada desde el derecho continental por Wielsch, quien afirma que difícilmente los autores de Wikipedia podrían calificar para la autoría en colaboración, según la legislación alemana.

Incluso si calificaran, ¿cuál sería la consecuencia con respecto al problema de la decisión de la autoridad? El régimen de administración de la autoría en colaboración en el derecho de autor es muy rígido. La decisión de publicar el trabajo colaborativo bajo una nueva licencia podría requerir permiso de todos los cocreadores (Wielsch, 2010: 203).

\subsection{OBRA COLECTIVA}

Obra colectiva es aquella «que sea producida por un grupo de autores, por iniciativa y bajo la orientación de una persona natural o jurídica que la coordine, divulgue y publique bajo su nombre» según la letra c) del artículo 5 de la LPI. A partir de esa definición, Herrera establece las características de la obra colectiva: «a) ser producida por un grupo de autores; b) debe ser efectuada por iniciativa y bajo la orientación de una persona natural o jurídica; c) coordinación, divulgación y publicación bajo el nombre de esta última persona» (I999: 28).

Llama la atención la pobreza regulativa de la obra colectiva en la LPI, la cual sólo se restringe a la definición del artículo 5 y a las remisiones que hace a obras específicas en el segundo párrafo del capítulo V. 
Tampoco la doctrina nacional se ha encargado de analizar este tipo de obras, tomando en cuenta las dudas que generan las expresiones «por iniciativa y bajo la orientación» y «coordinación, divulgación y publicación» en la definición del artículo 5. Éstas hacen referencia a la persona natural o jurídica a la que se le atribuye cierta titularidad sobre la obra colectiva; llámese organizador en el caso de las antologías, crestomatías, enciclopedias, diccionarios y otras compilaciones análogas, y empresa periodística en el caso de los diarios, revistas y otras publicaciones periódicas, según el artículo 24 de la LPI.

No podemos realizar un análisis sobre si las wikis pueden ser clasificadas como obras colectivas sin antes determinar a qué se refiere la ley con dichos conceptos. Para ello, recogeremos las normas de la obra colectiva existentes en las legislaciones española y francesa, que guardan ciertas similitudes con el concepto plasmado en la ley chilena. La Ley de Propiedad Intelectual de España la define en su artículo 8:

Se considera obra colectiva la creada por la iniciativa y bajo la coordinación de una persona natural o jurídica que la edita y divulga bajo su nombre y está constituida por la reunión de aportaciones de diferentes autores cuya contribución personal se funde en una creación única y autónoma, para la cual haya sido concebida sin que sea posible atribuir separadamente a cualquiera de ellos un derecho sobre el conjunto de la obra realizada. ${ }^{8}$

El Código de la Propiedad Intelectual de Francia, en su artículo Li I 32, define obra colectiva como:

La obra creada por iniciativa de una persona natural o jurídica que la edita, publica o divulga bajo su dirección y su nombre y en la que la contribución personal de los diversos autores que hayan participado en su elaboración se funde en el conjunto con vistas al cual está concebida sin que sea posible atribuir a cada uno de ellos un derecho diferente sobre el conjunto realizado. ${ }^{9}$

8. Real Decreto Legislativo I/I996, de I 2 de abril, por el que se aprueba el Texto Refundido de la Ley de Propiedad Intelectual, regularizando, aclarando y armonizando las disposiciones legales vigentes sobre la materia, I2 de abril de 1996.

9. Code de la Propriété Intellectuelle, I de julio de I992. La traducción es de Bataller (2002: 38 ). 
Primero, analicemos a qué se refiere el legislador por tener la iniciativa de la obra. Según el diccionario de la Real Academia Española (en adelante, «RAE»), iniciativa es aquello «que da principio a algo». Dicha palabra es utilizada en tres oportunidades en la LPI, en los artículos 5 letras c) y k) y 26; en estos tres casos hace referencia al organizador, al productor de fonogramas y al productor cinematográfico, respectivamente. Todas estas personas dan principio a la obra, aunque desde un rol más bien económico o técnico; la iniciativa, en estos casos, es la inversión que realizan dichas personas para la creación de la obra. Dicha concepción concuerda con el análisis desarrollado por Bataller, quien afirma que por iniciativa no se entiende necesariamente un impulso creativo a la obra, ya que es posible que la idea nazca de alguno de los coautores, los cuales «bus[can] posteriormente el manto protector de una persona (física o jurídica) con capacidad técnica para asumir su dirección y gestión» (2002: 54).

El siguiente concepto, la orientación de la obra, resulta más complejo de analizar, puesto que su uso en nuestra LPI se restringe a la disposición que analizamos, y no es mencionada en las leyes española y francesa. Sin embargo, basándonos en la redacción de la norma, podemos interpretar que, si por iniciativa de una persona se da origen a la producción de la obra, el estar bajo su orientación se refiere a su rol en la producción misma. Orientar, según la RAE, es «dirigir o encaminar a alguien o algo hacia un fin determinado». En nuestra opinión, la orientación implica establecer los límites para que los coautores realicen un trabajo que quede comprendido en el objetivo de la obra; en otras palabras, es una suerte de marco teórico de ésta. Por ejemplo, orientar sería definir que lo que se realizará es una enciclopedia y no una compilación de novelas.

Ahora debemos analizar qué concebimos por coordinación. Dado que en este tipo de obras se reúnen una serie de contribuciones independientes (artículos en el caso de las enciclopedias, noticias en los periódicos), cada una de las cuales tiene creadores diferentes, se necesita un ente que coordine a los distintos autores, ya que por lo general éstos no se entienden entre sí (y por tanto, no intervienen en las contribuciones de los demás). Tal como dice Bataller, la obra colectiva «es el resultado de un iter intelectual puesto en marcha y dirigido por alguien que se sobrepone a varios autores y otorga con su intervención una perspectiva de conjunto a las diversas aportaciones que cada uno de ellos pueda hacer» 
(2002: 52). De este modo, la coordinación encierra las tareas de buscar a los coautores, involucrarlos en el proyecto y corregir sus resultados (Bataller, 2002: 54-5).

La divulgación y publicación pueden englobarse en una misma tarea, la de dar a conocer la obra. En nuestra LPI la divulgación no está definida, a pesar de ser mencionada en varios artículos. La ley española la define como «toda expresión de la [obra] que, con el consentimiento del autor, la haga accesible por primera vez al público en cualquier forma» (artículo 4). La publicación es definida en el artículo 5 letra o) de nuestra LPI como «la oferta al público de la obra», mientras que la ley española dice que es «la divulgación que se realice mediante la puesta a disposición del público de un número de ejemplares de la obra» (artículo 4). Estas actividades, según la LPI, se hacen bajo el nombre del organizador. No ahondaremos en la divulgación y la publicación ya que, al ser éstas aplicables a todo tipo de obras, no son características que nos sirvan para establecer la esencia de la obra colectiva. ${ }^{10}$

Ahora debemos analizar si la wiki cumple con las características esenciales de la obra colectiva. La primera de ellas, respecto a que debe ser generada por múltiples autores, no es discutible, dado que es una herramienta esencialmente colaborativa.

Por el contrario, es controvertida la existencia de la segunda característica, que la obra sea realizada por iniciativa y bajo la orientación de la persona jurídica, en particular por la orientación. La primera de las condiciones, la iniciativa, no resulta problemática al ser comparable con la actividad del propietario de la wiki, quien da el impulso económico a ésta mediante la compra del hosting, el dominio y eventualmente del software.

Del modo que hemos entendido la orientación, como la tarea de dar un margen editorial a los aportes de los distintos coautores, su verificación dependerá de las funciones que tenga el propietario en la wiki.

La tercera característica, la coordinación, es la que nos genera más dudas, pues al igual que lo ocurrido con la orientación, su ejercicio dependerá del rol que tenga el propietario de la wiki en ésta. Creemos que los roles de orientación y coordinación pueden sintetizarse en el control del propietario de la wiki a los contenidos. Esto, porque el control no sólo

Io. Esperamos que el anterior examen de la obra colectiva sea un impulso a su estudio en nuestra legislación por parte de la doctrina del derecho de autor. 
opera de manera práctica, en la aceptación de usuarios y la revisión de los aportes de éstos (coordinación), sino que también de modo teórico, estableciendo parámetros de calidad y estándares editoriales (orientación).

Así, si utilizamos nuestra clasificación de las wikis basada en el control de contenidos, éstas pueden tener un administrador con control «activo» o "pasivo» de las contribuciones. Las wikis con control activo son aquellas en las que el propietario tiene la potestad para definir cuáles contenidos pueden ser desarrollados en la wiki, y cuáles no; esta figura es equivalente al editor de las obras colectivas clásicas, que ejerce las tareas de orientación y coordinación de los contenidos para que éstos no sean inarmónicos con el objetivo final de la obra.

Los paradigmas de wiki cuyo control es activo, y por ende, que podrían ser clasificados como obras colectivas, son las wikis «mixtas» (públicas de edición restringida) y «cerradas» (de acceso privado). Ejemplos de éstas son las wikis corporativas, las cuales son manejadas por empresas y en las que los usuarios son sus empleados, o Scholarpedia, donde los artículos son creados por expertos invitados y están sujetos a revisión por sus pares (peer review). En ambos casos, comparten con las obras colectivas clásicas la característica de que los usuarios son convocados por el organizador debido a sus conocimientos en cierta área, y éstos escriben sólo sobre los temas que dominan (cf. Doctorow, 20I2), los cuales se ajustan al estándar editorial de la wiki.

Sin embargo, el panorama es diferente en las wikis con control «pasivo» de los contenidos, dado que, si bien el propietario tiene la iniciativa de la obra (wiki), éste no ejerce las tareas de orientación y coordinación entre las contribuciones de los coautores.

El paradigma de wiki en la que existe un control pasivo de los contenidos es el de las wikis libres o propiamente tales. En éstas, los contribuyentes son libres para editar los temas que sean de su interés, y por lo general no existe censura previa (Reuveni, 2007: I8I I). En muchas wikis de este tipo, las funciones de orientación y coordinación están radicadas en la misma comunidad de usuarios, quienes ejercen tanto el control teórico a través del establecimiento de las políticas del sitio, que son definidas mediante votaciones y discusiones internas, así como el control práctico de los contenidos (revisión) o incluso de los usuarios que son bloqueados de la wiki por no cumplir con los estándares. 
Es cierto que los periódicos y enciclopedias tradicionales también representan una colección de artículos por una variedad de autores, pero en las wikis abiertas no existe la relación tradicional entre un editor y un autor. Los textos de wiki no son así directamente sujetos a la compulsión de lo comercializable (Ebersbach, Glaser y Heigl, 2005: 32).

En el caso de Wikipedia, la wiki más conocida, hay personas que plantean su eventual condición de obra colectiva basadas en la existencia de una persona jurídica, la Wikimedia Foundation, que según ellas administra la Wikipedia por su iniciativa y bajo su orientación, además de coordinar a sus múltiples usuarios (Maeztu, 2007). Como ya lo hemos dicho, no estamos de acuerdo con esta afirmación, por ser la Wikipedia un caso de wiki libre. Daremos una breve argumentación del caso específico.

Wikipedia y otros proyectos de wiki libre son, sin duda, generados por iniciativa de la Fundación Wikimedia. Sin embargo, la fundación no ejerce ni la orientación de la wiki, ni la coordinación entre los usuarios, la cual es realizada por la comunidad de usuarios de Wikipedia (Chen, 2010: 423 y ss). En sus "términos y condiciones de uso», la Fundación Wikimedia explicita que «actuamos solamente como un servicio de alojamiento, manteniendo la infraestructura técnica y organizativa que permite a nuestros usuarios construir los proyectos Wikimedia» y que «no ejercemos un rol editorial. Esto significa que generalmente no monitoreamos o editamos el contenido de los sitios web de los proyectos, y no ejercemos responsabilidad alguna por este contenido». Una sentencia reciente del Tribunal de Roma (Previti contra Wikimedia Foundation, Inc.), apoya esta tesis, al establecer que:

No se ha podido demostrar que, al administrar la enciclopedia Wikipedia, Wikimedia Foundation Inc. actúe diferente a un proveedor de alojamiento, esto quiere decir que es un sujeto que únicamente entrega alojamiento en sus servidores de información provista por los usuarios. ${ }^{11}$

En síntesis, las wikis de tipo libre no pueden ser clasificadas como obras colectivas al no darse todos los supuestos establecidos por la legislación chilena para la persona natural o jurídica que ejerce el rol de organizador. Como concluye Reuveni, las wikis y los demás modelos de

I I. Wikimedia Argentina, «Una victoria legal de Wikipedia en Italia», disponible en $<$ http://www.wikimedia.org.ar/20I3/06/26/una-victoria-legal-de-wikipedia-en-italia/>. 
contenido generado por usuarios son la «antítesis del modelo de producción jerárquico y corporativo presente a lo largo del siglo XX» (2007: I 808), o como nosotros diríamos, el modelo analógico manifestado en las obras colectivas tradicionales.

\section{OBRA COMPUESTA}

Además de la obra en coautoría analizada en el parágrafo anterior, existe un tercer tipo de obras en la que existe una pluralidad de autores (pluriautoría), pero que no es considerada obra en coautoría: la obra compuesta (Lipszyc, I993: I30). Dicha clasificación ha sido incorporada en algunas legislaciones de derecho continental, como Francia y España. Según el artículo 9 de la Ley de Propiedad Intelectual española, la obra compuesta es aquella «obra nueva que incorpore una obra preexistente sin la colaboración del autor de esta última, sin perjuicio de los derechos que a éste correspondan y de su necesaria autorización».

Si bien la LPI no se refiere de manera expresa a las obras compuestas, podría argumentarse que éstas son una especie de obra amparada en el derecho patrimonial de transformación, que consiste en «todo acto de modificación de la obra, comprendida su traducción, adaptación y cualquier otra variación en su forma de la que se derive una obra diferente» (artículo 5 letra w de la LPI).

Creemos, prima facie, que es difícil que una wiki pueda tener este carácter, ya que la independencia entre la obra preexistente y el autor de la obra compuesta traiciona el carácter colaborativo de las wikis. Esto, porque «el autor de la obra nueva debió obtener la autorización del autor de la obra antigua para poderla utilizar» (Rengifo, I997: I49), lo cual ralentizaría el proceso de producción de la wiki, al punto de hacerlo inviable.

Ello implica una barrera regulatoria de entrada a la participación en la creación mediante wiki, lo cual implica un perjuicio en general a las dinámicas de creación de conocimiento, y específicamente a la dinámica de la wiki que es esencialmente dependiente de la colaboración (Sereno, 20I0: 238 ).

Sin embargo, podría darse el caso que, en las wikis que recopilan contenido preexistente, exista alguna transformación de una obra bajo el 
dominio público, la cual no requeriría de un permiso para su utilización en una obra compuesta. Por ejemplo, en Wikisource la Declaración de Independencia de los Estados Unidos (obra preexistente) es traducida al español (obra compuesta). La traducción es, junto con la adaptación y la antología, una clase de obra compuesta según la legislación española (Colombet, 1997: 29).

Este último caso resulta excepcionalísimo, ya que por regla general en las wikis se crean contenidos originales; en las wikis libres los contenidos son generados «desde cero», y por tanto resultaría forzoso aplicarles este modelo de protección, puesto que no se verificaría uno de los requisitos esenciales de las obras compuestas, que es la obra preexistente.

\section{BASE DE DATOS}

El ideólogo de las wikis, Cunningham, las definió inicialmente como «la base de datos en línea más simple que pueda funcionar» (Cunningham y Leuf, 2004: I 5). Ello podría dar pie para considerar a la wiki como una base de datos, que a la postre la convertiría en un tipo de obra protegida por el derecho de autor.

El artículo 3 número I7 de la LPI incluye a las «compilaciones de datos o de otros materiales [...] que por razones de la selección o disposición de sus contenidos, constituyan creaciones de carácter intelectual», pero «no abarca los datos o materiales en sí mismos, y se entiende sin perjuicio de cualquier derecho de autor que subsista respecto de los datos o materiales contenidos en la compilación»; esta norma reproduce el artículo 5 del Tratado de la OMPI sobre Derecho de Autor (TODA). ${ }^{12}$

La Directiva 96/9/CE del Parlamento europeo y del Consejo del II de marzo de 1996 sobre la protección jurídica de las bases de datos de aquí en adelante, la Directiva- a su vez define a las bases de datos en su artículo I como «las recopilaciones de obras, de datos o de otros

I2. Tratado de la OMPI sobre Derecho de Autor; 20 de diciembre de I996. Su artículo 5 dice: «Las compilaciones de datos y de otros materiales, en cualquier forma, que por razones de la selección o de la disposición de sus contenidos constituyan creaciones de carácter intelectual, están protegidas como tales. Esa protección no abarca los datos o materiales en sí mismos y se entienden sin perjuicio de cualquier derecho de autor que subsista respecto de los datos o materiales contenidos en la compilación». 
elementos independientes dispuestos de manera sistemática o metódica y accesibles individualmente por medios electrónicos o de otra forma».

La Directiva entrega a las bases de datos dos clases de protección; un derecho de autor a las bases de datos que por «la selección o la disposición de su contenido constituyan una creación intelectual de su autor» (artículo 3 de la Directiva), y un derecho sui generis, que otorga un nivel de protección menor, puesto que no exige originalidad, «cuando la obtención, la verificación o la presentación de dicho contenido representen una inversión sustancial desde el punto de vista cuantitativo o cualitativo» (artículo 7 de la Directiva).

En virtud del Acuerdo sobre los Aspectos de los Derechos de Propiedad Intelectual relacionados con el Comercio (en adelante, "ADPIC») y del Convenio de Berna, los países contratantes no están obligados a otorgar esta protección sui generis a las bases de datos no originales (Ficsor, 2003: 250). De ahí que nuestra legislación no contenga una norma que reconozca expresamente dicha clase de protección, a diferencia de la Directiva europea o la Database Investment and Intellectual Property Antipiracy Act (H.R. 353 I) promulgada en los Estados Unidos en I996 (Schuster, I999: I4).

Phillips analiza la posibilidad de que la wiki sea una base de datos, tanto como obra protegida por derechos de autor, como obra protegida por derechos sui generis. En el primer caso, donde se exige una cuota de creatividad en la confección de una matriz de datos, se estaría creando una analogía con la recopilación de obras literarias; los contenidos serían propiedad de sus creadores (los usuarios), pero el propietario de la wiki tendría el derecho sobre la disposición sistemática de éstos. En el segundo caso, los derechos sui generis serían entregados al propietario por haber hecho una inversión sustancial para obtener, verificar y presentar el contenido (2008: 79I-3).

Este análisis cobra una enorme vigencia en la actualidad, considerando la creación de wikis con el propósito de generar bases de datos colaborativas; una de ellas es Wikidata, proyecto de la Wikimedia Foundation, cuya operación se inició en octubre de 20I2. Aprovechando esta tendencia, analizaremos si las wikis pueden ser protegidas tanto como bases de datos originales (protección por derechos de autor) como bases de datos no originales (protección sui generis). 


\subsection{BASES DE DATOS ORIGINALES}

Las bases de datos originales son «todas las compilaciones ya sea que existan en forma impresa o almacenada en un computador, que en razón de su original forma de selección o disposición de su contenido, puede constituir una obra protegida» (Schuster, I999: 4).

Considerar que las wikis libres sean bases de datos originales es dudoso, dadas las diferencias esenciales que presentan ambos formatos. Esto es algo advertido por Phillips, cuando plantea:

Una wiki, que es una compilación de contribuciones separadas, siempre podrá ser capaz de ser vista como una base de datos, a menos que las contribuciones sean realizadas en forma de pequeños y crecientes cambios e incrementos a una matriz de texto que no pueda ser calificada de 'independiente', dado que dichas contribuciones no pueden estar aisladas, y sólo tienen sentido o contexto por el contenido wiki que las sustenta (2008: 79I).

Las principales diferencias entre una wiki y una base de datos radican en el origen y disposición de los contenidos. El hecho de que la wiki no necesariamente tenga un contenido ya existente que se selecciona o dispone, sino que más bien éste se crea, modifica y edita en la misma plataforma, nos hace pensar que wiki es una idea que rebasa el concepto de base de datos. Además, la estructura de las wikis libres es de contenido orgánico y acceso abierto, mientras que las bases de datos son rígidas y de acceso restringido.

La originalidad de las bases de datos dice relación con el «hecho de que la selección o disposición del contenido de la base de datos constituya una labor de creación intelectual propia del autor» (considerando I 5 del preámbulo de la Directiva). En las wikis libres, en cambio, al no existir una intervención activa del propietario de la wiki en la disposición de los contenidos, no se cumpliría este supuesto.

\subsection{BASES DE DATOS NO ORIGINALES}

Las bases de datos que no cumplen con el presupuesto de originalidad podrían recibir protección sui generis en cuanto hayan requerido una inversión sustancial para obtener, verificar y presentar el contenido. En 
el caso de las wikis, «el proveedor de una base de datos basada en wiki podrá reclamar el derecho sui generis si logra demostrar que ha hecho una inversión sustancial en la provisión de la plataforma» (Schmitz, 20I 2: I2).

Esta protección sui generis podría ser aplicada al software y la interfaz que soporta la wiki, como por ejemplo MediaWiki, puesto que éste requiere de un esfuerzo patrimonial, tanto en su desarrollo como en su mantención. De este modo se protegería la inversión monetaria del propietario de la wiki para recopilar los contenidos generados por los usuarios.

Sin embargo, si aplicamos a la wiki cualquiera de los tipos de bases de datos, nos encontramos con un problema fundamental: la protección sui generis que les otorga el derecho de autor en algunas legislaciones atiende a la estructura de la base de datos, no a los contenidos de ésta, los cuales reciben protección por el derecho de autor de manera independiente (Schuster, I999: I2). Los usuarios de la wiki no podrían invocar titularidad sobre esta protección sui generis.

En resumen, las wikis pueden considerarse bases de datos en cuanto a su estructura (software), pudiendo ésta recibir protección sui generis, pero no en cuanto a los contenidos generados por los usuarios, que es lo relevante a efectos de este estudio, y que por lo tanto dejaría a los contenidos de las wikis libres — por el momento- en una suerte de desregulación por el derecho de autor.

\section{ANÁLISIS PARCIAL}

En esta sección hemos podido comprobar que la doctrina no ha logrado de manera categórica establecer el tipo de figura jurídica a la que corresponden las wikis según el derecho de autor. Sólo se ha podido dar una solución parcial al problema, pudiendo homologar ciertas wikis —las de tipo cerradas y mixtas- a la obra colectiva, mientras que se podría otorgar una protección sui generis a las wikis.

Sin embargo, las wikis libres, que encarnan el paradigma más cercano al concepto original de wiki, no engarzan con ninguna de las clasificaciones de obra que se han desarrollado en la legislación, la doctrina y la jurisprudencia del derecho de autor. 
En síntesis, según los paradigmas de wikis, el panorama general quedaría de la siguiente manera:

Cuadro 1. Calificación jurídica de los paradigmas de wiki

\begin{tabular}{|l|l|l|}
\hline & Contenido de la wiki & Software que lo soporta \\
\hline Libres & No aplicable & \\
\hline Mixtas & Protección como obra colectiva & $\begin{array}{l}\text { Protección sui generis } \\
\text { (base de datos no original) }\end{array}$ \\
\hline Cerradas & \\
\hline
\end{tabular}

\section{LA ENTRADA DE WIKI COMO OBRA}

Como explicábamos al comienzo, la wiki se compone por páginas conocidas como «entradas», en cada una de las cuales se contienen los contenidos de la wiki. Cada vez que un usuario edita dicha página, genera una versión de la entrada. Mientras que la versión más reciente aparece desplegada al ingresar al contenido con un dominio de Internet fijo (a lo cual llamaremos entrada propiamente tal), las versiones anteriores pueden ser accedidas a través del historial de la entrada. En otras palabras, la entrada propiamente tal es un contenido que muta en el tiempo, mientras que la versión de la entrada permanece estática y no puede ser editada sin generar una nueva versión (Sereno, 2010: 223).

A continuación estudiaremos la factibilidad de proteger mediante el derecho de autor tanto la entrada propiamente tal como la versión de la entrada.

\section{LA ENTRADA DE WIKI PROPIAMENTE TAL}

La entrada de wiki en sí misma no es susceptible de ser protegida por el derecho de autor, dada la imposibilidad de ser considerada una obra, atendiendo a su carácter dinámico (Simone, 20I I). La facilidad con que pueden ser modificados los contenidos de una entrada podría implicar ver una obra totalmente distinta cada vez que se ingresa a una página de wiki. En este sentido, Sereno dice:

En virtud del principio según el cual el derecho de autor protege formas de expresión y no ideas o procedimientos y de una observación de- 
tallada del funcionamiento de la wiki, se concluye que la forma escrita de expresión de ideas, es decir, la obra literaria, objeto protegible por el derecho de autor, se encuentra en las versiones de la entrada, y no en esta última, cuya naturaleza se asemeja más al proceso de creación (incremental) de conocimiento (2010: 222).

Nos remitimos a lo anteriormente dicho respecto a las dificultades de dar protección a los contenidos de una wiki, ya que, atendida su estructura, no se permite delimitar fácilmente las ediciones en cada entrada, sino que los aportes se muestran como un todo único.

Por otro lado, este tipo de protección sería injusta, ya que un usuario que ha aportado la parte más sustancial de la entrada, ya sea en términos cuantitativos o cualitativos, tendría los mismos derechos sobre el contenido que otro usuario que sólo haya realizado ediciones menores. Así, serían coautores los usuarios que aportaron, por ejemplo, el 99\% del texto de la entrada y el que corrigió el I \%.

Considerar a la entrada de wiki como obra sería homologarla a una obra en colaboración, pero como ya hemos visto, es poco probable que en las wikis libres se cumpla el requisito de que todos los coautores aporten una cantidad considerable de contenidos originales a la obra.

\section{LAS VERSIONES DE LA ENTRADA DE WIKI}

Los contenidos de la wiki serían objeto de protección por el derecho de autor - explica Sereno- en tanto versiones de la entrada individual, es decir, las páginas web que muestran un estado temporal de la entrada de wiki. Según el colombiano,

una versión de la entrada es una forma expresiva literaria estática, temporalmente acabada, claramente determinada, por regla general original (creada independientemente y con un mínimo de creatividad), es decir, una obra literaria fija (2010: 222).

Para desarrollar esta idea, Sereno se vale de los conceptos de obra originaria y obra derivada. La LPI define obra originaria como «aquella que es primigénitamente creada» (artículo 5 letra h de la LPI), mientras que obra derivada es «aquella que resulte de la adaptación, traducción u otra transformación de una obra originaria, siempre que constituya una creación autónoma» (artículo 5 letra i de la LPI). 
En el caso de la wiki, la primera versión de un artículo sería una obra original, mientras que las versiones posteriores serían obras independientes, pero derivadas de ésta. La titularidad de los derechos estaría radicada en los usuarios que crearon las versiones del artículo, nunca en el propietario de la wiki (Sereno, 2010: 225-6).

La solución de Sereno parece ser la más sensata que nos puede otorgar el derecho de autor clásico. Se hace cargo de la dificultad para diferenciar las contribuciones de los usuarios de la manera más práctica posible, mediante el acceso al historial, donde cada versión está individualizada por su autor y el tiempo de su creación.

No obstante, la anterior propuesta deja muchas interrogantes, que intentaremos repasar en lo siguiente. El principal de los problemas está relacionado a la exigencia al autor de una obra derivada de contar con el permiso del autor de la obra originaria para poder usarla, o de lo contrario está infringiendo sus derechos (Sereno, 2010: 23I). ¿Es esto posible en las wikis libres? Al menos a simple vista no nos parece que así sea. El requisito de solicitar autorización al titular de la última versión de una entrada va en contra el espíritu mismo de la wiki, que es la colaboración, ya que la acción de editar se vuelve demasiado engorrosa. Esto implicaría no sólo un incentivo a la infracción de derechos de autor, sino que también la desprotección de las nuevas versiones desautorizadas, frenando la creación.

Por ejemplo, en los casos en que el último editor es anónimo, es decir, cuando aparece representado por su dirección IP, sería (casi) imposible pedir autorización, lo que terminaría por desincentivar el crecimiento de los contenidos de la wiki.

Además, la solicitud de autorización peca de odiosa. Piénselo bien: supongamos que usted redacta un artículo, y al día siguiente entra a la wiki para ampliarlo, pero resulta que otro usuario hizo una edición para cambiar un punto por una coma, ¿le parecería adecuado tener que pedirle permiso a esa persona para realizar las mejoras necesarias?

Una forma de evitar el problema de la necesidad de una autorización por parte del usuario anterior es el uso de licencias libres, como GNU GLP (GNU General Public License) o Creative Commons, las cuales tienen como objeto complementar las leyes de derecho de autor y flexibilizar los derechos patrimoniales que emanan de éstas, con el fin de facilitar el uso y adaptación de obras ajenas. 
En muchas wikis, incluyendo Wikipedia, los usuarios que aportan nuevos contenidos deben aceptar términos de uso, que incluyen el licenciamiento libre de esos contenidos. Así, tanto usuarios registrados como anónimos aceptan la posterior utilización y modificación de sus aportes. Esto resulta coherente con el hecho que los usuarios saben, o es razonable que sepan, que al generar contenidos en una wiki éstos pueden ser modificados posteriormente, ya que es de la naturaleza de la plataforma que así sea.

Sin embargo, la aplicación de licencias puede llevar aparejados nuevos problemas. Uno de ellos es la elección de la licencia, que puede ser realizada tanto por el administrador - en los casos de las wikis cerradas y mixtas - como por la comunidad de usuarios, en el caso de las wikis abiertas. La aplicación de una licencia puede provocar oposición por una parte de los usuarios que no estén conformes con las implicancias de la licencia (por ser demasiado permisivas o demasiado restrictivas); de este modo, una licencia podría llegar a ser también un factor de desincentivo para el crecimiento de una wiki.

Un ejemplo concreto de esta problemática de elección de una licencia fue lo ocurrido con Wikipedia en 2009, cuando por votación de la comunidad se adoptó la actual licencia Creative Commons «ReconocimientoCompartirIgual» (Attribution-ShareAlike, by-sa), reemplazando la GNU GFDL que había usado desde 200 I. Esta decisión estuvo antecedida por un intenso debate respecto a las consecuencias de ese cambio; mientras que los usuarios favorables a la licencia «CC by-sa» argumentaban que las restricciones de la GFDL eran contradictorias con la cultura libre, los detractores al cambio esgrimían como principales argumentos que la nueva licencia, al permitir el uso comercial, permitía a su vez el lucro a costa del trabajo de los voluntarios (Roessing, 2010: 38-40).

En cuanto al objeto de nuestro análisis, si bien las licencias libres dan una respuesta práctica al problema de la regulación de las wikis, ésta sería parcial ya que sólo se remite a los derechos patrimoniales sobre la obra, pero nada resuelve respecto a un eventual ejercicio de derechos morales.

Finalmente, las licencias libres no pueden ser consideradas un elemento de la esencia de las wikis, y difícilmente podrían ser elementos de la naturaleza, dada su voluntariedad; de este modo, un administrador o una comunidad de usuarios podría decidir legítimamente no licenciar 
los contenidos de una wiki mediante una de estas licencias voluntarias, y «reservarse todos los derechos», u omitir una decisión al respecto, con lo que regresaríamos a una incertidumbre regulatoria.

A continuación analizaremos otras problemáticas que provocaría considerar a las versiones de las entradas de wiki como obras derivadas.

En primer lugar, Sereno (2010: 225-6) dice que la entrada originaria debe tener una cuota de originalidad que le permita ser una obra como tal, siendo muy excepcionales los casos que no cumplirían con ese requisito, como los enlaces a páginas inexistentes (ejemplo por lo demás errado, ya que las páginas que no se han creado simplemente no son páginas).

Nosotros podríamos dar casos más precisos de entradas de wiki que dudosamente podrían ser calificadas de «originales» en el sentido de la doctrina tradicional. Uno de ellos es la entrada que ha sido traducida desde una wiki en otro idioma, ¿es una obra originaria por el sólo hecho de ser la primera versión del artículo? No, es obra derivada (o una obra compuesta según la legislación española).

Otro caso son las redirecciones, páginas que reconducen automáticamente a otras con el objeto de acaparar los nombres posibles para un mismo tema; por ejemplo, en Wikipedia «Dictadura de Pinochet» y «Gobierno Militar (Chile)» redirigen a «Régimen Militar (Chile)», dando una solución salomónica a un tema controversial. ¿Son las redirecciones obras susceptibles de protección? Podemos decir categóricamente que no lo son. Y, por lo demás, este tipo de páginas está lejos de ser una excepción; en agosto de 20 Io había 724.007 entradas y I.I78.238 redirecciones en la Wikipedia en español. ${ }^{13}$

En segundo lugar, este modo de autoría da por supuesto que el autor primario aportará con una parte sustancial del contenido, pero, ¿qué sucedería si quien inicia una entrada sólo escribiera una frase, mientras que los autores de las versiones posteriores añadieran una gran cantidad de contenido a esa entrada? Pongamos un ejemplo.

Un usuario, Alicia, crea una entrada de wiki sobre Chile, escribiendo «Chile es un país de Sudamérica». Posteriormente Bernardo agrega veinte

I3. Wikipedia, Contribuyentes de Wikipedia: Informes automáticos/Número de páginas por espacio de nombres, disponible en <http://es.wikipedia.org/wiki/Wikipedia:Informes_ automáticos/Número_de_páginas_por_espacio_de_nombres>. 
párrafos sobre la geografía, demografía, historia, política y economía de Chile. En tercer lugar, Carlos corrige una falta de ortografía en una de las secciones. Según esta forma de autoría, Alicia tendría la autoría originaria del artículo, mientras que Bernardo y Carlos serían autores derivados, que deben solicitar permiso a los anteriores para poder hacer su contribución. ¿Qué pasaría si Alicia se negara a que Bernardo agregue la información? Una vez más, se desincentivaría la creación de contenidos.

Según la propuesta de algunos usuarios de wiki, debería existir, en vez de una autoría derivada, una «autoría temporal» que recaería en el último editor de la entrada, quien asumiría la responsabilidad por la información contenida. Esta concepción, además de no tener sustento jurídico, es igualmente inconsistente con las técnicas de la plataforma wiki, ya que un usuario que hizo un aporte menor podría ser el autor temporal del artículo (en el ejemplo anterior, Carlos, que sólo hizo una corrección ortográfica, sería el autor temporal del artículo sobre Chile).

En síntesis, las versiones de una entrada de wiki, si bien teóricamente podrían protegerse bajo los conceptos de obra originaria y obra derivada, en la práctica presentan dificultades para su aplicación, fundamentalmente por la necesidad de solicitar autorización a los autores anteriores, y porque las licencias libres no son elementos esenciales de estas plataformas.

\section{LAS SECCIONES DE LA ENTRADA DE WIKI}

Además de plantear si es posible otorgar autoría en las entradas de wikis como un todo y en las versiones de aquellas, existe un tercer criterio que atiende a la disposición espacial de los contenidos al interior de una entrada. Según éste, cada usuario sería autor de la porción del artículo que creó.

La entrada de una wiki que haya adoptado el «modo hilado», en el cual cada usuario firma sus contribuciones, podría sustentar este modo de protección. Sin embargo, ello no es válido para las wikis en «modo documento», ya que este formato no fomenta la identificación de las contribuciones de cada usuario, sino que las presenta como un todo indivisible.

Esta concepción de autoría no se podría aplicar, por ello, a la mayoría de las wikis libres, puesto que por regla general ellas han adoptado el 
«modo documento», ya que, como hemos explicado, es más coherente con el espíritu colaborativo de la herramienta wiki.

Por ejemplo, en Wikipedia todas las entradas están estructuradas en el «modo documento», y la misma wiki establece expresamente que los artículos no pueden firmarse. ${ }^{14} \mathrm{El}$ «modo hilado» se restringe a las páginas de discusión de los artículos de Wikipedia, en donde los usuarios se limitan a opinar sobre el contenido de la entrada.

\section{CONCLUSIONES}

Como hemos podido demostrar en este artículo, sólo dos de los tres principales formatos de wiki logran ser encasillados en los arquetipos jurídicos de obras que ha desarrollado el derecho de autor. De este modo, las wikis cerradas y las wikis mixtas, que se caracterizan por el control activo de los contenidos por parte del propietario de la wiki, pueden ser clasificadas como obras colectivas. El software que da estructura a la wiki es análogo a las bases de datos, que en algunas legislaciones reciben una protección sui generis por la inversión sustancial para su desarrollo.

Sin embargo, las wikis libres, que encarnan el paradigma más cercano al concepto de wiki, dada su estructura dinámica y abierta, no pueden asimilarse a los tipos de obra que establece el derecho de autor tradicional, considerándolas tanto obras en sí mismas, como también compilaciones de obras, que serían las entradas o las versiones temporales de éstas.

Si las wikis libres son obras bajo el concepto del Convenio de Berna, pero no pueden ser clasificadas como las obras típicas del derecho de autor —sin el riesgo, lógicamente, de desnaturalizar el concepto de wiki-, la conclusión más lógica para su regulación y protección estaría en la creación de un nuevo tipo de obras multiautorales, que permita tanto a las wikis libres, como a otras plataformas colaborativas similares (como

I 4. Véase Wikipedia, Contribuyentes de Wikipedia: Acerca de firmar artículos, disponible en <http://es.wikipedia.org/wiki/Wikipedia:Acerca_de_firmar_art \% $3 \%$ ADculos >. La página dice: «Es una norma de Wikipedia nunca firmar los artículos, porque consideramos que es una falta de respeto al resto de los wikipedistas. Todas las colaboraciones quedan reflejadas junto con su autor en el historial del artículo. Se recuerda que los artículos de Wikipedia son propiedad de todos sus autores y están licenciados bajo las licencias CC-BY-SA y GFDL, por lo que pueden ser modificados por cualquiera que respete las políticas». 
Google Drive o Editorially), poder subsanar las trabas que actualmente el derecho de autor pone a la dinámica colaborativa.

No obstante, dicha solución es lenta y poco viable, en el sentido que implicaría un desarrollo doctrinal en la materia aparejado de una reforma legal de los estatutos de propiedad intelectual. Además, como bien explica Sereno, «las reformas internacionales y legales recientes han apuntado a una dirección opuesta: la revigorización del derecho patrimonial de autor en el entorno digital y de mecanismos paralelos que dan soporte a esos derechos en la era digital» (2010: 243).

Una segunda solución es el ya analizado uso de las licencias libres. Es una práctica común que las wikis adopten estas licencias con el objeto de eliminar la barrera de entrada que el derecho de autor implica en el uso y edición de estas plataformas; en otras palabras, evita el requerimiento de autorización previa por parte del autor (o los autores) para generar nuevos contenidos.

Sin embargo, las licencias libres sólo resuelven el problema práctico, pero no el doctrinal, ya que no permiten calificar jurídicamente a la wiki. Esto, porque dichas licencias son complementarias al derecho de autor, y no buscan reemplazarlo. Además, la aplicación de licencias libres es voluntaria, y sin una base legal (o quizás, jurisprudencial), no podrían presumirse aplicables a estas plataformas como un elemento, a lo menos, de la naturaleza de las wikis.

El debate está planteado respecto a las wikis, pero el tema no se agota en ellas. Creemos fundamental que el derecho de autor asuma de una manera concluyente el devenir de la tecnología, y avance más allá de la concepción analógica de las obras que la doctrina tradicional persiste en mantener como un dogma irrefutable.

\section{REFERENCIAS}

BAtaller R., Enric (2002). La obra colectiva. Valencia: Tirant lo Blanch. Black, Peter, Hayden Delaney y Brian Fitzgerald (2007). «Legal issues for wikis: The challenge of user-generated and peer-produces knowledge, content and culture». eLaw Journal, I4 (I): 245-282.

Charman, Suw y Michael Holloway (2006). "Copyright in a collaborative age». M/C Journal, 9 (2). Disponible en <http://journal.mediaculture.org.au/0605/02-charmanholloway.php >. 
Chen, Shun-Ling (2010). «Self-governing online communities in Web 2.0: Privacy, anonymity and accountability in Wikipedia». Albany Law Journal of Science and Technology, 20: 42 I-432.

Chon, Margaret (I996). «New wine bursting from Old Bottles: Collaborative Internet art, joint works, and entrepreneurship». Oregon Law Review Spring, 1996: 257-276.

Colombet, Claude (1997). Grandes principios del derecho de autor y los derechos conexos en el mundo: Estudio de derecho comparado. Tercera edición. Unesco/Cindoc/CINDOC.

Cunningham, Ward y Bo Leuf (2004). The wiki way: Quick collaboration on the web. Segunda edición. Boston: Addison-Wesley.

Doctorow, Cory (2012). "Why Philip Roth needs a secondary source». The Guardian, I9 septiembre. Disponible en <http://www. guardian.co.uk/technology/20 I 2/sep/ı 9/why-philip-roth-needs-secondary-sources.

Ebersbach, Anja, Markus Glaser y Richard Heigl (2005). Wiki: Web collaboration. Berlín: Springer.

FARley, Martin (2007). "Web 2.0, wikis, and the IP community». Journal of Intellectual Property Law \& Practice, 2 (4).

FICSOR, Mihály (2003). Guía sobre los tratados de derecho de autor y derechos conexos administrados por la OMPI. Ginebra: Organización Mundial de la Propiedad Intelectual.

García, Rosa (2005). El derecho de autor en Internet. Madrid: Colex.

GARON, Jon (20IO). «Wiki authorship, social media, and the curatorial audience». Harvard Journal of Sports and Entertainment Law, I (I): 95-I 44 .

George, Carlisle E. y Jackie Scerri (2007). «Web 2.0 and user-generated content: Legal challenges in the new frontier». Journal of Information, Law and Technology, 2007 (2).

Herrera, Dina (i999). Propiedad intelectual derechos de autor: Ley I 7.336 y sus modificaciones. Santiago: Jurídica.

LAMB, Brian (2004). «Wide open spaces: Wikis, ready or not». Educause Review, 39 (5): 36-48.

Lipszyc, Delia (1993). "Titulares del derecho de autor». En Derechos de autor y derechos conexos. París, Bogotá, Buenos Aires: Ediciones Unesco-Cerlalc- Zavalía. 
Maeztu, David (2007). «¿Qué es, jurídicamente, la Wikipedia?». Disponible en <http://derechoynormas.blogspot.com/2007/I 2/qu-es-juridicamente-la-wikipedia.html>.

Phillips, Jeremy (2008). "Authorship, ownership, wikiship: Copyright in the twenty-first century». Journal of Intellectual Property Law \& Practice, 3 (I 2): 788-798.

Rengifo, Ernesto (1997). Propiedad intelectual: El moderno derecho de autor. Segunda edición. Bogotá: Universidad Externado de Colombia.

Reuveni, Erez (2007). "Authorship in the age of the conducer». Copyright Society of the USA, 54 (2I8): I8OI-I 859.

Roessing, Thomas (2010). "Authorship in Wikipedia: Legal requirements, community opinions, and technical boundaries». Masaryk University Journal of Law and Technology, 4 (I): 35-45.

Schmitz, Sandra (2012). Protection of the content of a 'wiki' database under the european database directive. Luxemburgo: Interdisciplinary Center for Security, Reliability and Trust.

Schuster, Santiago (I999). «Los derechos de autor en la supercarretera de la información: Regulación de las bases de datos originales y no originales». En Segundo curso taller de formación de profesores en materia de propiedad intelectual. Ciudad de México: OMPI.

Sereno, Juan (20IO). "Contenido generado por usuarios (UGC), wikis y derecho de autor». La Propiedad Inmaterial, I4: 206-260.

Simone, Daniela (20I I). "Copyright or copyleft? Wikipedia as a turning point for authorship». Disponible en <http://www.law.depaul.edu/ centers_institutes/ciplit/ipsc20 I I/pdf/Powerpoints/SimoneD_Powerpoint.pdf>.

Snake-Beings, Emit (2010). The construction of Karen Karnak: The multi-author-function. Thesis for the degree of Masters of Arts Screen and Media. Nueva Zelanda, The University of Waikato. Disponible en<http://researchcommons.waikato.ac.nz/handle/I0289/4327>.

Wielsch, Dan (20I0). "Governance of massive multiauthor collaboration». Journal of Intellectual Property, Information Technology and E-Commerce Law, I (2): 96-го8. 


\section{SOBRE EL AUTOR}

Marco Correa Pérez es egresado de la Facultad de Derecho de la Universidad de Chile, miembro fundador y director de la corporación Wikimedia Chile. El presente artículo es una versión parcial de su memoria para optar al grado de Licenciado en Ciencias Jurídicas y Sociales de esa misma casa de estudios. Su correo electrónico es <marco.correa@ wikimediachile.cl>.

Este trabajo fue recibido el 28 de agosto de 2013 y aprobado el I 9 de noviembre de 2013 . 